\title{
ON THE INCLINATION DEPENDENCE OF EXOPLANET PHASE SIGNATURES
}

\author{
Stephen R. Kane and Dawn M. Gelino \\ NASA Exoplanet Science Institute, Caltech, MS 100-22, 770 South Wilson Avenue, Pasadena, CA 91125, USA; skane@ipac.caltech.edu \\ Received 2011 December 2; accepted 2011 January 5; published 2011 February 10
}

\begin{abstract}
Improved photometric sensitivity from space-based telescopes has enabled the detection of phase variations for a small sample of hot Jupiters. However, exoplanets in highly eccentric orbits present unique opportunities to study the effects of drastically changing incident flux on the upper atmospheres of giant planets. Here we expand upon previous studies of phase functions for these planets at optical wavelengths by investigating the effects of orbital inclination on the flux ratio as it interacts with the other effects induced by orbital eccentricity. We determine optimal orbital inclinations for maximum flux ratios and combine these calculations with those of projected separation for application to coronagraphic observations. These are applied to several of the known exoplanets which may serve as potential targets in current and future coronagraph experiments.
\end{abstract}

Key words: planetary systems - techniques: photometric

\section{INTRODUCTION}

The changing phases of an exoplanet as it orbits the host star have long been considered as a means for their detection and characterization (Charbonneau et al. 1999; Leigh et al. 2003). The relation between giant planet atmospheres and phase curves has been described in detail by Sudarsky et al. (2005) and Kane \& Gelino (2010, hereafter KG10). However, the relatively small flux ratio of the planet to the host star has presented a major hindrance to the realization of such detections. A new era of optical and infrared (IR) telescopes is enabling phase detections in a manner which was previously inaccessible from the ground.

Attempts to detect phase signatures have primarily been for transiting planets. Examples of observed phase variations in the IR include HD 189733b (Knutson et al. 2009b) and HD $149026 b$ (Knutson et al. 2009a). Examples in the optical include Kepler observations of HAT-P-7b (Welsh et al. 2010) and phase variations detected in the light curve of CoRoT-1b (Snellen et al. 2009). Phase variation detection of non-transiting planets has been restricted to hot Jupiters, including $v$ And $\mathrm{b}$ (Harrington et al. 2006) and HD 179949b (Cowan et al. 2007). There has also been recent observational evidence for the detection of phase variations by the non-transiting planet HD 46375 b by Gaulme et al. (2010). Planets in eccentric orbits, such as HD 17156b (Barbieri et al. 2007) and HD 80606b (Laughlin et al. 2009), will produce relatively high phase amplitudes during a brief interval.

The orbital inclination is usually preferred to be edge-on for optimal detection of phase variations since this ensures that full phase will occur along the line of sight. However, this is not necessarily the case depending upon the eccentricity and periastron argument of the orbit, and indeed there are cases where the maximum flux ratio occurs when the orbit is faceon. Even though the radial velocity technique is biased toward the detection of edge-on orbits, since these have a larger radial velocity semi-amplitude, certainly this is not always the case. Astrometric studies such as that performed by Han et al. (2001) have shown that there are a variety of orbital inclinations as expected, and so it is prudent to consider this for attempted phase detections.

Here, we present a thorough exploration of orbital parameter space and the impact upon flux ratios for eccentric planets. The main causes of degeneracy are the periastron argument and inclination for a given eccentricity. We further calculate projected separations at apastron as a function of inclination and determine their correspondence with maximum flux ratio locations. With all orbital elements considered, one may then use the phase locations of maximum flux ratio and maximum projected separation to quantify the suitability for follow-up observations.

\section{FLUX RATIO COMPONENTS}

In this section, we outline the theoretical framework which will be used throughout the remainder of the paper. This formalism has been used extensively by Collier Cameron et al. (2002) and more recently by Rodler et al. (2010). We refer the reader to KG10 for a more detailed description of the particular formalism used here.

The flux ratio of a planet with radius $R_{p}$ to the host star is defined as

$$
\epsilon(\alpha, \lambda) \equiv \frac{f_{p}(\alpha, \lambda)}{f_{\star}(\lambda)}=A_{g}(\lambda) g(\alpha, \lambda) \frac{R_{p}^{2}}{r^{2}},
$$

where the flux is measured at wavelength $\lambda$. This flux ratio consists of three major components; the geometric albedo $A_{g}(\lambda)$, the phase function $g(\alpha, \lambda)$, and the inverse-square relation to the star-planet separation $r$. Each of these are briefly described below in the context of eccentric orbits. Note that the observed flux ratio from an exoplanet is wavelength dependent in that the atmospheric composition drives the scattering properties and thus the shape of the geometric albedo and phase function. As was the case with KG10, we confine our study to optical wavelengths centered on $550 \mathrm{~nm}$.

Atmospheric models have shown that there is a dependence of the geometric albedo of giant planets on the semi-major axis of the orbit (Sudarsky et al. 2000, 2005; Cahoy et al. 2010). To account for this, we use the analytic function described by KG10 which was in turn derived from the models of Sudarsky et al. (2005). This function results in a time dependence of the albedo as the strong irradiation of the atmospheres of giant planets removes reflective condensates from the upper atmospheres during periastron passage. This function can have a dramatic effect in dampening the flux ratio at small star-planet separations. 
The phase angle $\alpha$ is defined to be zero when the planet is at superior conjunction and is described by

$$
\cos \alpha=\sin (\omega+f),
$$

where $\omega$ is the argument of periastron and $f$ is the true anomaly. Thus, in terms of orbital parameters, minimum phase occurs when $\omega+f=90^{\circ}$ and maximum phase occurs when $\omega+f=270^{\circ}$.

The exact nature of a planetary phase function depends upon the assumptions regarding the scattering properties of the atmosphere. Rather than assuming isotropic scattering (Lambert sphere), we adopt the empirically derived phase function of Hilton (1992) which is based upon observations of Jupiter and Venus and incorporates substantially more back-scattering due to cloud-covering. This approach contains a correction to the planetary visual magnitude of the form

$$
\Delta m(\alpha)=0.09\left(\alpha / 100^{\circ}\right)+2.39\left(\alpha / 100^{\circ}\right)^{2}-0.65\left(\alpha / 100^{\circ}\right)^{3},
$$

leading to a phase function given by

$$
g(\alpha)=10^{-0.4 \Delta m(\alpha)},
$$

which is used throughout the remainder of this paper.

As shown by Sudarsky et al. (2005) and KG10, the maximum flux ratio does not necessarily occur at the zero phase angle for a non-circular orbit. This is because the star-planet separation $r$ is constantly changing and is given by

$$
r=\frac{a\left(1-e^{2}\right)}{1+e \cos f},
$$

where $a$ is the semi-major axis and $e$ is the orbital eccentricity. The $R_{p}^{2} / r^{2}$ component of Equation (1) becomes dominant for highly eccentric orbits, which is especially important when the orbital inclination is considered.

\section{ORBITAL INCLINATION}

To add the effect of the inclination angle to the phase function, the phase angle (Equation (2)) is modified as follows:

$$
\cos \alpha=\sin (\omega+f) \sin i .
$$

At first and third quarters $\left(\alpha=90^{\circ}\right.$ and $\left.\alpha=270^{\circ}\right)$, the flux ratio is completely independent of the inclination angle. However, at all other phase angles there arises a complex pattern of flux ratios from the inclination and argument of periastron. Here, we describe these dependences.

\subsection{Edge-on/Face-on Orbits}

The two cases worth considering first are the extremes of edge-on $\left(i=90^{\circ}\right)$ and face-on $\left(i=0^{\circ}\right)$ orbits. As noted earlier, the radial velocity method is biased toward the detection of edge-on orbits due to the increase in the semi-amplitude of the signal. In addition, most of the planets monitored for phase signatures are known to transit. Thus, the case of edge-on orbits is currently the dominant form of investigated systems. This guarantees the observability of both zero phase and full phase, the contribution of which to the flux ratio depends upon the star-planet separation at $\alpha=0^{\circ}$.

The case of face-on orbits means that the phase function becomes completely flat since only half phase will be visible at any one time. Thus, the flux ratio is completely determined by the eccentricity of the orbit which drives both the star-planet separation and the changing albedo of the upper atmosphere.

\subsection{Generalized Orientation}

Beyond the cases of edge-on and face-on orbits, the interaction of inclination and periastron argument becomes more complex. Figure 1 demonstrates this for four eccentric orbits, each with fixed periastron arguments, which are inclined from edge-on to face-on. Within the range of $45^{\circ} \lesssim \omega \lesssim 135^{\circ}$, the flux ratio actually increases with decreasing orbital inclination as the day side of the planet becomes more visible where the planet is the hottest, most noticeable for the case of $\omega=90^{\circ}$. Outside of this regime, the peak flux from the planet generally declines as the inclination increases and thus the access to the full phase diminishes.

\subsection{Peak Flux Ratio Maps}

A further level of detail to the description of inclination dependence may be added by calculating the peak flux ratio for the full range of inclinations $\left(0^{\circ}<i<90^{\circ}\right)$ and periastron arguments $\left(0^{\circ}<\omega<360^{\circ}\right)$ for a given period and eccentricity. The resulting intensity maps then show the optimal orbital configuration for detection and how the distribution of peak flux ratios smoothly varies with these configurations.

Shown in Figure 2 are two such examples of these intensity maps for eccentricities of 0.2 and 0.8 . In each case, the strongest flux ratio occurs where the full phase of the planet coincides with the smallest star-planet separation $\left(i=90^{\circ}, \omega=270^{\circ}\right)$. However, notice the interaction which occurs where $\omega=90^{\circ}$. The changing peak planetary flux along this line of the intensity maps is due to the competing components of the phase function and star-planet separation as the planet moves from a crescent phase near periastron to the full phase of the planet at apastron. For highly eccentric orbits, the planet flux at $\omega=90^{\circ}$ becomes the brightest when the orbit is viewed face-on since the flux ratio is dominated by the star-planet separation.

\section{PROJECTED SEPARATION}

The star-planet projected separation is a component which will influence the target selection for coronagraph experiments for the direct detection of the reflected planetary flux. Beichman et al. (2010) tabulate the inner working angle of selected future ground-based imaging instruments which range from 0.03 to 0.17, compared to 0'.035 to 0.'850 for James Webb Space Telescope (JWST) instruments. The angular projected separation of the planet from the star is given by

$$
\Delta \theta=\frac{r}{d}\left(\cos ^{2}(\omega+f)+\sin ^{2}(\omega+f) \cos ^{2} i\right)^{\frac{1}{2}},
$$

where $d$ is the star-observer distance. Here we utilize the analysis of the previous section to determine the orbital location of maximum flux ratio for a subset of the known exoplanets. In Table 1, we report the true anomaly $f$ (angle between the direction of periapsis and the current position of the planet in the orbit), flux ratio $\epsilon$, and projected separation $\Delta \theta$ at this location for the most eccentric exoplanets. We additionally report if the maximum flux ratio occurs for an edge-on or face-on orientation. Notice that for face-on orbits, the true anomaly is zero at this location since the flux is completely driven by the event of periastron passage.

There is a bias against optimal separation since maximum flux naturally occurs when the planet is closest to the star. This is most severe for edge-on orbits, such as HD 80606b where the angular separation is $\sim 0^{\prime \prime}$ at this location. For this reason, the 

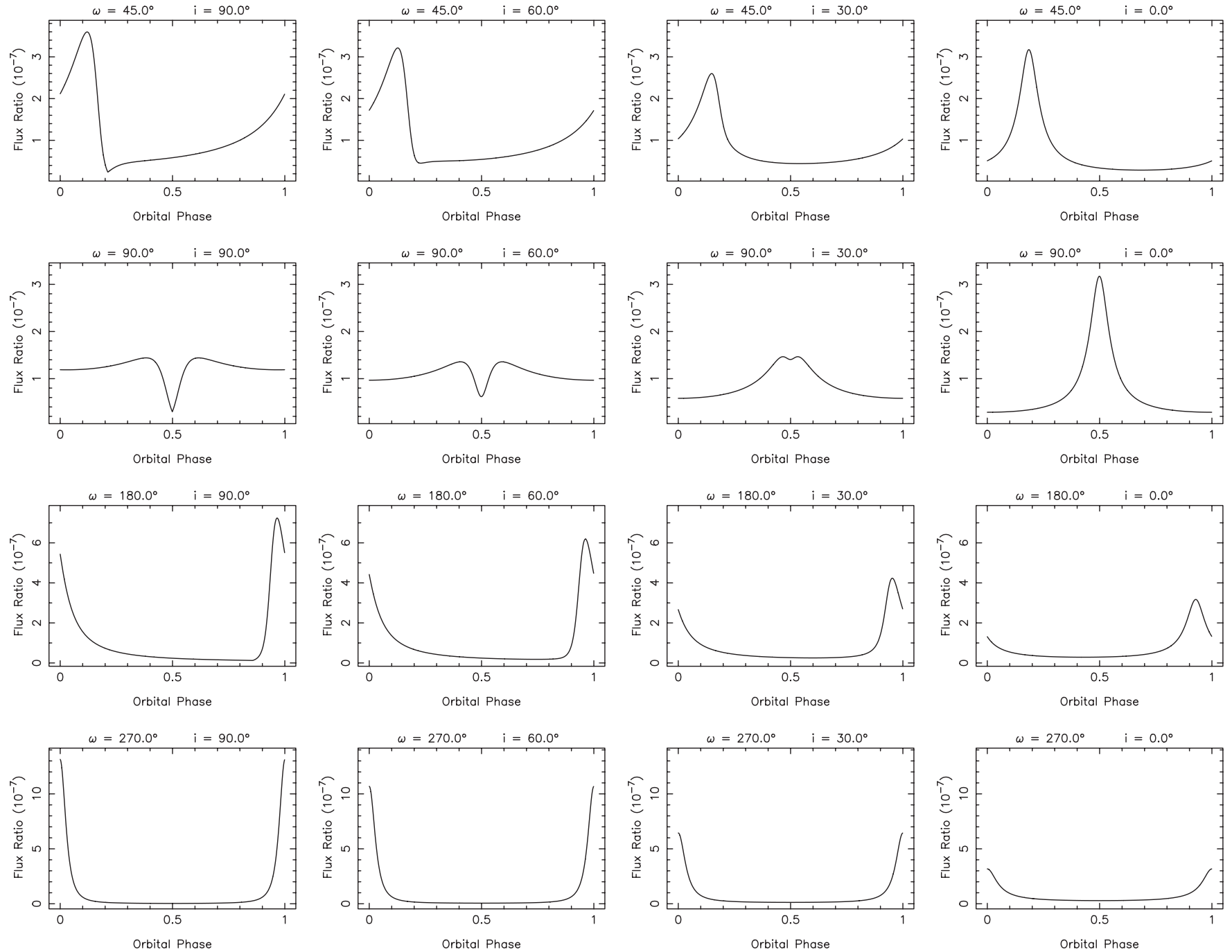

Figure 1. Calculated flux ratios for various orbital configurations with $e=0.6$ and $P=100$ days. Each row represents a specific periastron argument with inclinations starting from edge-on $\left(i=90^{\circ}\right)$ and progressing to face-on $\left(i=0^{\circ}\right)$.

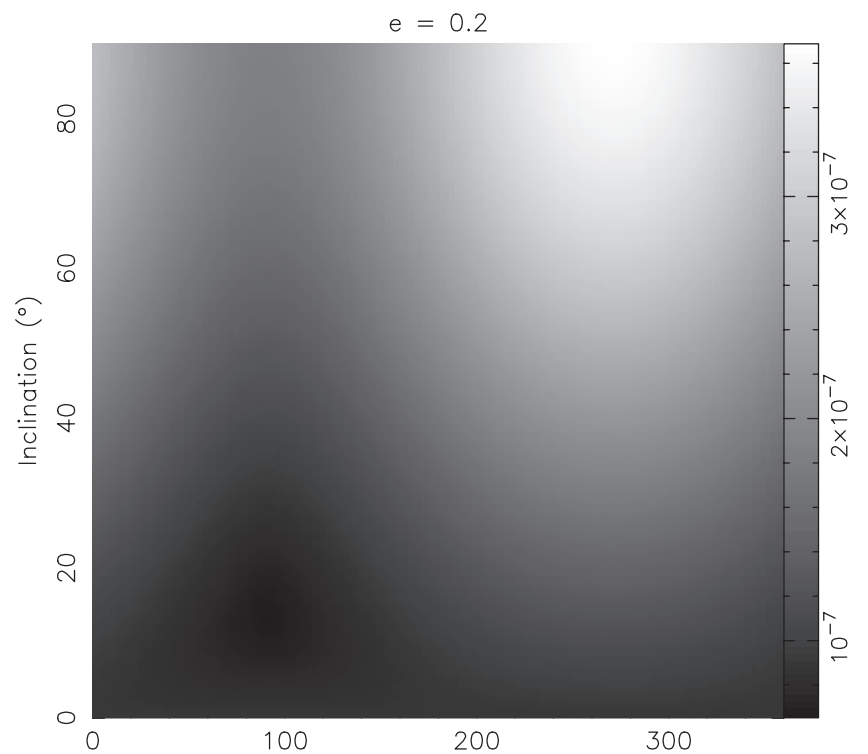

Argument of Periastron $\left({ }^{\circ}\right)$

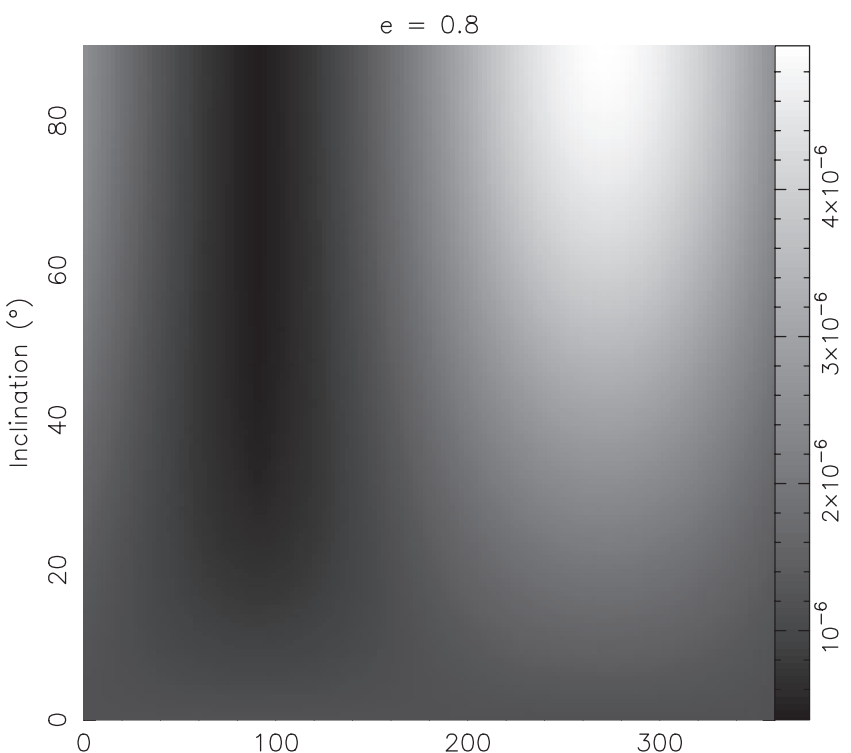

Argument of Periastron $\left({ }^{\circ}\right)$

Figure 2. Intensity maps for a $P=100$ days planet with $e=0.2$ (left) and $e=0.8$ (right). These maps show the peak flux ratio values for the full range of inclinations and periastron arguments. For $e=0.2$, the minimum peak flux ratio occurs where $\omega=90^{\circ}$ and $i=13^{\circ}$. 


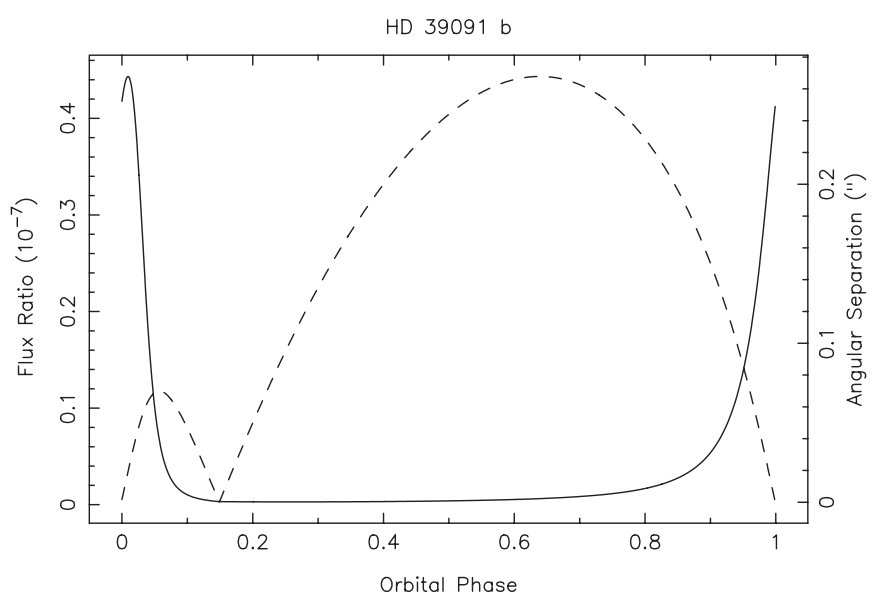

Figure 3. Flux ratio (solid line) and star-planet angular separation (dashed line) for HD 39091b, assuming an edge-on orbit.

location of maximum flux ratio preferentially corresponds to the location of minimum angular separation. Consider the case of HD 39091b (Figure 3) where the peak flux arises from an edge-on orientation. The peak flux ratio corresponds to a $00^{\prime \prime} 02$ separation, whereas the planet reaches a separation of $\sim 0$ '.26 during the entire phase. By matching the angular separations above the resolution criteria of the coronagraph, one can choose the optimal targets for monitoring during the predicted orbital phase of peak flux ratio.

\section{EXOPLANET CHARACTERIZATION}

The main targets for which phase signature detection will be attempted will likely be those for which a planet is already known to be present. In this case, the purpose of the observations is characterization rather than discovery. As shown in Equation (1), the flux ratio is a function of the geometric albedo $A_{g}$, the periastron argument $\omega$, the orbital inclination $i$, and the planetary radius $R_{p}$. The challenge of disentangling those components from the phase shape and amplitude will vary depending upon what is already known for that system.

If the planet is known to transit, then one can measure $i, \omega$, and $R_{p}$. One can thus determine $A_{g}$ which, along with models of the planetary structure based upon the size and mass, can constrain the properties of the atmosphere. If however the planet does not transit, then we can estimate the radius of the planet from the measured mass using theoretical models, such as those of Bodenheimer et al. (2003) and Fortney et al. (2007), for which there will be associated uncertainties depending upon factors such as the age of the planet and the assumed core model. In some cases, astrometric measurements of the host star can be performed, such as those carried out by Han et al. (2001). This will have the simultaneous result of resolving the inclination of the orbit, thus breaking the degeneracy of the flux ratio with the periastron argument, and determining the true mass of the secondary companion. For non-transiting planets discovered using the radial velocity method, one has the advantage of a strong bias toward bright host stars relative to those discovered using the transit method. The increase in signal to noise will be a great asset for an appropriate instrument to exploit, as described in Section 6.

Possible contamination of the phase signature at photometric passbands by internal heating of the planet is worth considering. If indeed the thermal signature of the planet is dominated by internal heating rather than incident flux from the host star,
Table 1

$f$ and $\Delta \theta$ at Maximum $\epsilon$

\begin{tabular}{|c|c|c|c|c|c|c|}
\hline Planet & $P(\mathrm{~d})$ & $e$ & $\omega\left(^{\circ}\right)$ & $f\left({ }^{\circ}\right)$ & $\epsilon\left(10^{-5}\right)$ & $\Delta \theta\left(^{\prime \prime}\right)$ \\
\hline HD 80606 b & 111.4 & 0.93 & 300.6 & $339.7^{e o}$ & 3.80295 & 0.00003 \\
\hline HD 20782 b & 585.9 & 0.93 & 147.0 & $65.4^{e o}$ & 0.09461 & 0.00336 \\
\hline HD $4113 b$ & 526.6 & 0.90 & 317.7 & $330.1^{e o}$ & 0.19394 & 0.00091 \\
\hline HD 156846 b & 359.5 & 0.85 & 52.2 & $0.0^{f o}$ & 0.03101 & 0.00359 \\
\hline $\mathrm{HD} 45350 \mathrm{~b}$ & 963.6 & 0.78 & 343.4 & $311.8^{e o}$ & 0.01603 & 0.00441 \\
\hline HD $30562 b$ & 1157.0 & 0.76 & 81.0 & $0.0^{f o}$ & 0.00380 & 0.02126 \\
\hline HD 20868 b & 380.9 & 0.75 & 356.2 & $306.1^{e o}$ & 0.03875 & 0.00332 \\
\hline HD $37605 \mathrm{~b}$ & 54.2 & 0.74 & 211.6 & $36.8^{e o}$ & 0.56082 & 0.00063 \\
\hline HD $222582 \mathrm{~b}$ & 572.4 & 0.73 & 319.0 & $326.9^{e o}$ & 0.02634 & 0.00258 \\
\hline HD $2039 \mathrm{~b}$ & 1120.0 & 0.71 & 344.1 & $308.3^{e o}$ & 0.00912 & 0.00277 \\
\hline iota Dra b & 511.1 & 0.71 & 91.6 & $0.0^{f o}$ & 0.00771 & 0.01182 \\
\hline HD $96167 \mathrm{~b}$ & 498.9 & 0.71 & 285.0 & $348.6^{e o}$ & 0.02805 & 0.00029 \\
\hline HD $86264 b$ & 1475.0 & 0.70 & 306.0 & $333.1^{e o}$ & 0.00 & 0.00206 \\
\hline HAT-P-13 c & 428.5 & 0.69 & 176.7 & $61.3^{e o}$ & 0.01674 & 0.00123 \\
\hline HD 159868 b & 986.0 & 0.69 & 97.0 & $0.0^{f o}$ & 0.00328 & 0.01054 \\
\hline HD $17156 b$ & 21.2 & 0.68 & 121.9 & $0.0^{f o}$ & 0.33167 & 0.00068 \\
\hline 16 Cyg B b & 798.5 & 0.68 & 85.8 & $0.0^{\text {fo }}$ & 0.00417 & 0.02497 \\
\hline HD 89744 b & 256.8 & 0.67 & 195.1 & $49.5^{e o}$ & 0.02977 & 0.00380 \\
\hline HD $39091 \mathrm{~b}$ & 2151.0 & 0.64 & 330.2 & $315.4^{e o}$ & 0.00429 & 0.02000 \\
\hline HD 131664 b & 1951.0 & 0.64 & 149.7 & $81.7^{e o}$ & 0.00236 & 0.01908 \\
\hline HD 74156 b & 51.6 & 0.63 & 176.5 & $59.6^{e o}$ & 0.15667 & 0.00116 \\
\hline HD 154672 b & 163.9 & 0.61 & 265.0 & $4.7^{e o}$ & 0.07147 & 0.00002 \\
\hline HD $171028 b$ & 538.0 & 0.61 & 305.0 & $334.2^{e o}$ & 0.01717 & 0.00076 \\
\hline HD $16175 b$ & 990.0 & 0.60 & 222.0 & $36.4^{e o}$ & 0.00744 & 0.00317 \\
\hline HD $3651 \mathrm{~b}$ & 62.2 & 0.60 & 245.5 & $17.5^{e o}$ & 0.24309 & 0.00133 \\
\hline HD $175167 b$ & 1290.0 & 0.54 & 342.0 & $305.2^{e o}$ & 0.00450 & 0.00570 \\
\hline HIP 2247 b & 655.6 & 0.54 & 112.2 & $119.9^{e o}$ & 0.00354 & 0.02233 \\
\hline HD 190228 b & 1136.1 & 0.53 & 101.2 & $0.0^{\text {fo }}$ & 0.00127 & 0.01983 \\
\hline HD 108147 b & 10.9 & 0.53 & 308.0 & $333.3^{e o}$ & 1.36640 & 0.00026 \\
\hline CoRoT-10 b & 13.2 & 0.53 & 218.9 & $35.3^{e o}$ & 0.01872 & 0.00004 \\
\hline HD $87883 b$ & 2754.0 & 0.53 & 291.0 & $343.2^{e o}$ & 0.00328 & 0.00682 \\
\hline HD 142022 b & 1928.0 & 0.53 & 170.0 & $72.2^{e o}$ & 0.00252 & 0.02467 \\
\hline HD $168443 b$ & 58.1 & 0.53 & 172.9 & $65.4^{e o}$ & 0.09881 & 0.00244 \\
\hline HD $81040 b$ & 1001.7 & 0.53 & 81.3 & $0.0^{f o}$ & 0.00186 & 0.02773 \\
\hline HIP 5158 b & 345.7 & 0.52 & 252.0 & $14.2^{e o}$ & 0.02411 & 0.00070 \\
\hline HD $4203 \mathrm{~b}$ & 431.9 & 0.52 & 329.1 & $315.8^{e o}$ & 0.01272 & 0.00207 \\
\hline HD $217107 \mathrm{c}$ & 4270.0 & 0.52 & 198.6 & $49.2^{e o}$ & 0.00117 & 0.05555 \\
\hline HAT-P-2 b & 5.6 & 0.52 & 185.2 & $57.0^{e o}$ & 2.55946 & 0.00016 \\
\hline HD $1237 \mathrm{~b}$ & 133.7 & 0.51 & 290.7 & $344.2^{e o}$ & 0.06497 & 0.00120 \\
\hline HD 142415 b & 386.3 & 0.50 & 255.0 & $12.3^{e o}$ & 0.01697 & 0.00074 \\
\hline
\end{tabular}

Note. ${ }^{e o}$ indicates edge-on orbit, ${ }^{f o}$ indicates face-on orbit.

the signature will maintain a constant offset of the predicted photometric signature. The self-luminous properties of the planet will be a strong function of the age of the system, and will be restricted to planets whose age is $(<1 \mathrm{Gyr})$. Current radial velocity surveys choose targets based upon stability as well as spectral line features and thus prefer $\mathrm{F}-\mathrm{G}-\mathrm{K}$ main-sequence stars where activity is known to be reduced (Wright 2005). Thus, targets drawn from the radial velocity discoveries will preferentially be of an age whereby thermal contamination of the optical phase signature will be minimal.

\section{FEASIBILITY REQUIREMENTS}

In this section, we briefly outline requirements which will influence the detection of the previously described phase signatures.

\subsection{Instrumentation}

The detection of the phase amplitudes described here presents a significant challenge to instrumentation requirements. As 
mentioned in Section 1, optical phase variations have been detected in Kepler photometry. However, the vast majority of Kepler targets are relatively faint and so not ideally suited toward characterization with current follow-up capabilities. Conversely, planets discovered through radial velocities have bright host stars by comparison.

The instrumentation requirement for the successful detection of the signatures shown in Table 1 is photometry with an accuracy of $\sim 10^{-6}$. The necessary stability of this precision over long timescales depends upon the fraction of the orbital phase over which the largest change in phase amplitude occurs, described in detail by KG10. The photometer for the Kepler mission, for example, is designed to achieve high-precision photometry over the $6.5 \mathrm{hr}$ window of a transit, but is not designed for long-term stability over the lifetime of the mission (Borucki et al. 2010). Fortunately, the orbits of the radial velocity planets are well understood in most cases and so we can accurately predict not just the amplitude of the predicted phase signature but also the phase and times of maximum and minimum flux ratios. This knowledge will help to distinguish the phase signatures from instrumental drift effects.

From the ground, the challenges are more substantial since one also needs to contend with the offsets from night-to-night variations. Future generation telescopes will provide opportunities to achieve very high precision, such as the European Extremely Large Telescope, the Thirty Meter Telescope, and the Giant Magellan Telescope. It has been demonstrated by Colón et al. (2010) that precision photometry of $<0.05 \%$ can be achieved with large telescopes through the use of narrowband filters.

The phase variation due to the planetary orbit is the main observable we have discussed here, but these calculations can be combined with projected separations for use with coronagraphs, as described in Section 4. Ground-based coronagraph experiments, such as that being pursued at Palomar (Hinkley et al. 2011), are rapidly improving in instrument efficiency and the suppression of speckle noise. Simulations of these coronagraphs indicate that long-term stability may indeed be possible (Beichman et al. 2010), though this may only be feasible in the short term at the needed precision for young self-luminous planets. Space-based instruments, such as the planned Lyot coronagraph on NIRCam for the JWST, may be able to achieve phase detections for a sample of the most favorable targets, though in this case the instrument is optimized toward young planets around late-type stars.

\subsection{Stellar Variability}

At the level of photometric precision required here, it is important to consider the level of intrinsic stellar variability. An analysis of Kepler data by Ciardi et al. (2011) found that most dwarf stars are stable down to the precision of the Kepler spacecraft, with $\mathrm{G}$ dwarfs being the most stable of the studied spectral types. The well-known orbital parameters of the radial velocity target stars will aid in separating the signals of planetary phase from that of the host star variability. Additionally, most of the known exoplanet host stars have been well characterized through extensive ground-based photometry and spectroscopy.

The main cause of photometric variability in $\mathrm{F}-\mathrm{G}-\mathrm{K}$ stars is starspots and rotation, as verified by the Kepler variability study performed by Basri et al. (2011). The effects of starspots on exoplanet detection have been previously studied, such as the work of Makarov et al. (2009). Since the distribution of the starspot periodicity is related to the rotation rates of these stars, the detection of short-period planets (such as most of the known transiting planets) is relatively unaffected by these variations. However, there will inevitably be cases where the orbital period of the planet is close to the rotation period of the star, which is generally in the range of 10-40 days for radial velocity host stars (Simpson et al. 2010). In such cases, the peaks in the power spectrum from a Fourier analysis of the photometry may separate to a degree where the starspot variability can be isolated from the phase signature. It should be noted that disentangling these signals may substantially increase the required observing time.

\section{CONCLUSIONS}

The push toward characterizing the atmospheres of exoplanets will rapidly expand once further direct measurements of atmospheric albedos and thermal properties become possible. Current space telescopes are already detecting exoplanet phase variations in the optical (e.g., Kepler) and the IR (e.g., Spitzer). One approach to optimizing searches for phase variations in known eccentric systems is the refinement of orbital parameters through radial velocity measurements (Kane et al. 2009). The peak flux ratio maps may then be used to constrain the detectability of each system and the location of the peak intensity with respect to the projected separation, particularly important for proposed coronagraph missions such as the SpectroPolarimetric Imaging and Characterization of Exo-planetary Systems (SPICES) mission. More imminently, Kepler will soon detect transiting long-period ( $P>100$ days) planets, where the bias will be toward eccentric orbits since those have a higher probability of transiting (Kane \& von Braun 2008). In this case, the results of the presented work may be utilized to predict flux ratios for edge-on orbits as an independent measurement of the periastron argument. The faintness of these host stars will provide many instrumentation and observing challenges which make known radial velocity planets more attractive targets despite their unknown orbital inclination. Even so, as more science results are released by the Kepler mission, the study of photometric phase variations of long-period planets will become an increasingly relevant endeavor in the characterization of exoplanets.

The authors thank David Ciardi and Warren Skidmore for several useful discussions. We thank the anonymous referee whose comments greatly improved the quality of the paper. This research has made use of the Exoplanet Orbit Database and the Exoplanet Data Explorer at http://exoplanets.org.

\section{REFERENCES}

Barbieri, M., et al. 2007, A\&A, 476, L13

Basri, G., et al. 2011, AJ, 141, 20

Beichman, C. A., et al. 2010, PASP, 122, 162

Bodenheimer, P., Laughlin, G., \& Lin, D. N. C. 2003, ApJ, 592, 555

Borucki, W., et al. 2010, Science, 327, 977

Cahoy, K. L., Marley, M. S., \& Fortney, J. J. 2010, ApJ, 724, 189

Charbonneau, D., Noyes, R. W., Korzennik, S. G., Nisenson, P., Jha, S., Vogt, S. S., \& Kibrick, R. I. 1999, ApJ, 522, L145

Ciardi, D. R., von Braun, K., Bryden, G., van Eyken, J., Howell, S. B., Kane, S. R., Plavchan, P., \& Stauffer, J. R. 2011, AJ, submitted

Collier Cameron, A., Horne, K., Penny, A., \& Leigh, C. 2002, MNRAS, 330 187

Colón, K. D., Ford, E. B., Lee, B., Mahadevan, S., \& Blake, C. H. 2010, MNRAS, 408, 1494

Cowan, N. B., Agol, E., \& Charbonneau, D. 2007, MNRAS, 379, 641

Fortney, J. J., Marley, M. S., \& Barnes, J. W. 2007, ApJ, 659, 1661

Gaulme, P., et al. 2010, A\&A, 518, L153 
Han, I., Black, D. C., \& Gatewood, G. 2001, ApJ, 548, L57

Harrington, J., Hansen, B. M., Luszcz, S. H., Seager, S., Deming, D., Menou, K., Cho, J. Y.-K., \& Richardson, L. J. 2006, Science, 314, 623

Hilton, J. L. 1992, in Explanatory Supplement to the Astronomical Almanac, ed. P. K. Seidelmann (Mill Valley, CA: Univ. Science Books), 383

Hinkley, S., et al. 2011, PASP, in press

Kane, S. R., \& Gelino, D. M. 2010, ApJ, 724, 818

Kane, S. R., Mahadevan, S., von Braun, K., Laughlin, G., \& Ciardi, D. R. 2009, PASP, 121, 1386

Kane, S. R., \& von Braun, K. 2008, ApJ, 689, 492

Knutson, H. A., Charbonneau, D., Cowan, N. B., Fortney, J. J., Showman, A. P., Agol, E., \& Henry, G. W. 2009a, ApJ, 703, 769

Knutson, H. A., et al. 2009b, ApJ, 690, 822

Laughlin, G., Deming, D., Langton, J., Kasen, D., Vogt, S., Butler, P., Rivera, E., \& Meschiari, S. 2009, Nature, 457, 562
Leigh, C., Collier Cameron, A., Horne, K., Penny, A., \& James, D. 2003, MNRAS, 344, 1271

Makarov, V. V., Beichman, C. A., Catanzarite, J. H., Fischer, D. A., Lebreton, J., Malbet, F., \& Shao, M. 2009, ApJ, 707, L73

Rodler, F., Kürster, M., \& Henning, T. 2010, A\&A, 514, A23

Simpson, E. K., Baliunas, S. L., Henry, G. W., \& Watson, C. A. 2010, MNRAS, 408, 1666

Snellen, I. A. G., de Mooij, E. J. W., \& Albrecht, S. 2009, Nature, 459 543

Sudarsky, D., Burrows, A., Hubeny, I., \& Li, A. 2005, ApJ, 627, 520

Sudarsky, D., Burrows, A., \& Pinto, P. 2000, ApJ, 538, 885

Welsh, W. F., Orosz, J. A., Seager, S., Fortney, J. J., Jenkins, J., Rowe, J. F., Koch, D., \& Borucki, W. J. 2010, ApJ, 713, L145

Wright, J. T. 2005, PASP, 117, 657 